RESEARCH NEEDS IN CEMENT-BASED WASTE FORMS* CONF-901105--23

E. W. McDaniel

R. D. Spence

O. K. Tallent

Chemical Technology Division

Oak Ridge National Laboratory

P. O. Box 2003

Oak Ridge, Tennessee 37831-7273

DE91 004387

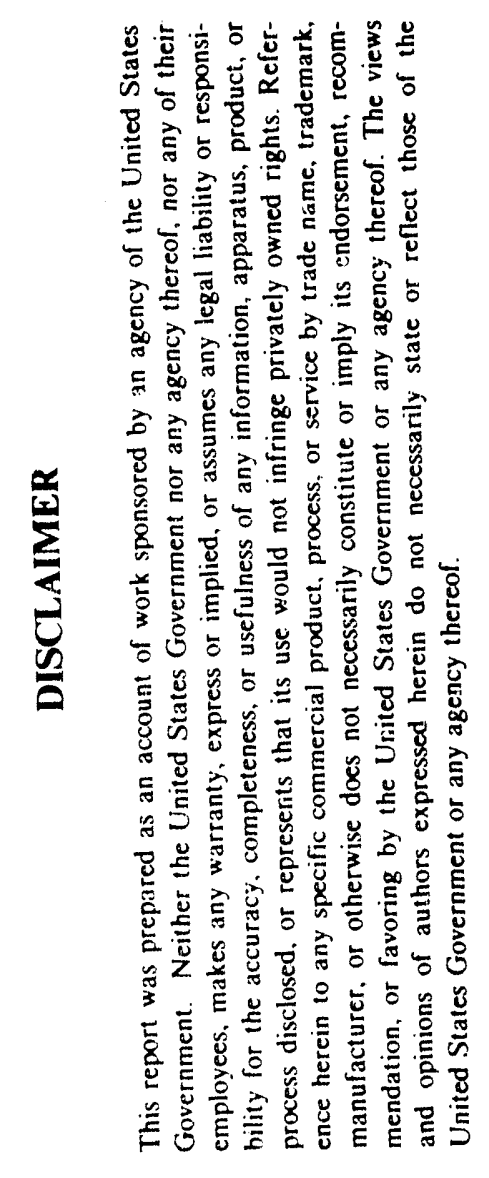

For presentation at the

1990 Fall Meeting of the Materials Research Society,

XIV International Symposium on the Scientific

Basis for Nuclear Waste Management

Boston, Massachusetts

November 26-29, 1990

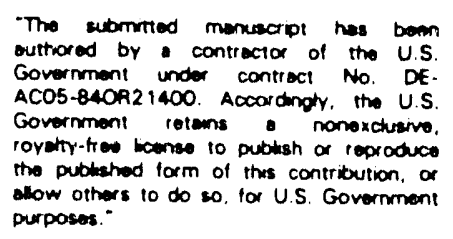

Research sponsored by the Westinghouse Hanford Company and performed at the Oak Ridge National Lahoratory, operated hy Martin Marictia Energy Systems, Inc., for the U.S. Department of Energy under contract DE-AC05-OR21400. 


\title{
RESEARCH NEEDS IN CEMENT-BASED WASTE FORMS
}

\author{
E W. MCDANIEL, R. D. SPENCE, AND O. K. TALLENT \\ Oak Ridge National Laboratory, P.O. Box 2003, Oak Ridge, TN $37831-7273$
}

\begin{abstract}
Cement-based waste forms are one of the most widely used waste disposal options, yet definitive knowledge of the fate of the waste species inside the waste form is lacking. A fundamental understanding of the chemistry and microstructure of the waste forms would lead to a better understanding of the mass transfer of the waste species, more confidence in predicting and extrapolating waste form performance, and design of hetter waste forms. Better and cheaper leach tests would lead to quicker and more cost effective screening of waste form alternatives. In addition, assessment of durability may be important to predicting waste form performance in the field.
\end{abstract}

\section{INTRODUCTION}

Environmental and ecological concerns constitute some of the most important technical concerns of our time. These concerns are without question national and international in scope. Radioactive and industrial waste management are included as a significant part of the concerns. Immobilization of waste in cement-based materials is probably the most widely accepted radioactive and industrial waste management method. Thus, it is extremely important that immobilization mechanisms in cement-based waste forms be better understood not only for credibility purposes thut for better utilization of the method as well. It should be noted that the research needs discussed in this report are from the perspective of investigators working in applied waste management areas, while the proposed investigations are fundamental or basic. Details as to experimental methods and tools to be used in achieving the objectives of the proposed are research beyond the scope of this paper and are better filled in by others.

In broad terms, the research topics discussed are (1) correlation of cemient-based waste form physical properties to performance, (2) waste-form fundamental chemistry and microstructure, and (3) product performance lesting.

An appreciation of the research needs for industrial radioactive waste requires background information on the substances in the waste in addition to the radioisotopes. The wastes in the form of liquids or slurries usually contain high concentrations of nitrate, hydroxide, sodium, and aluminum with lesser amounts of chloride, fluoride, sulfide, and potassium. Importanlly, the wastes also frequently 
contain low but significant concentrations of toxic substances such as mercury, sclenium, lead, arsenic, and other inorganic and organic toxic substances.

Blend materials vary but may include different types of cement, ny ash, ground blast furnace slags, and different clays. Small amounts of additives, set retarders for example, may also be added. Standard procedures have been developed for formulation mixing, curing, and testing.

\section{CORRELATION OF PHYSICAL PROPERTIES TO PERFORMANCE}

Correlation of physical properties to product performance is closely related to the Adsorption Equilibrium topic discussed further on in this report. A number of investigators have taken the viewpoint that the performance of cement-based waste forms is largely a function of the physical propertics of the waste form. Product properties such as density, pore size, pore volume, internal surface area, tortuosity, and others have been studied. Tentative formulae to mathematically relate such properties to diffusivity and electrical conductivity and ultimately to product durability and leachability have been derived. The importance of this work can readily be seen when it is considered that large fractions of the waste species in the system may be immobilized on pore surfaces or in pore liquids and further that species initially elsewhere in the waste-form matrix may eventually diffuse to pore surfaces and into nore liquids. Continued work in this investigative area is important and is stressed.

\section{FUNDAMENTAL CHEMISTRY AND MICROSTRUCTURE}

Much of the performance testing and measurement of bulk physical properties of these waste forms can be done, and sometimes is done, with little or no knowledge of the fate of the waste species inside the waste form. Essentially, the waste form is a "black box" that releases the waste species at a measured rate. This lack of fundamental knowledge makes it difficult to extrapolate from one application to another and can lead to spectacular failures. Some of the questions are: What is the fate of the waste species inside the waste form? Do they become part of the binder matrix? Are they retained in a relatively insoluble chemical environment? Are they located interstitially among the binder

particles? What is their effect on the binder reactions? What is the role of oxygen potential and valence state? Are there more than one species? Does solid diffusion occur or is mass transfer hasically pore diffusion hampered by surface interaction? Is it more important to have a high surface area or a low porosity?

Workers are not totally lgnorant of the chemistry and microstructure of their waste forms. Some work has been done in this area and this knowledge has been put to good use in some applications, but 
this work is usually not definitive, and the applications are usually of a speculative nature. More fundamental knowledge will give a better understanding of why a waste form "works," how one application can he extrapolated to another, how to design better waste forms, how to predict the performance of waste forms, and how to extrapolate the performance of a waste form into the future with confidence.

\section{Adsorption cquilibrium}

The complexities and intricacies involved in immobilization mechanisms in cement-based waste forms become apparent when adsorption equilibria in the system are considered. These equilibria are, 10 a large extent, considered to be functionally related to the above discussed physical properties. The amount of material adsorbed in the system is considered to be closely related to the waste product internal surface arca, which, in turn, is a function of waste-form pore-size distribution, pore volume, and density.

Chemical compositions and chemical concentrations on pore surfaces and in pore liquids are other factors which may affect adsorption equilibria. Fluoride on pore surfaces, for example, may tend to decrease desorption from the surfaces into pore liquids, while nitrate in pore liquids may tend to accelerate desorption of some species. Complexing agents, $\mathrm{pH}$, formation of low solubility compounds (metal oxy-nitrates, etc.), and phenomenon such as common ion effects are all factors which can effect adsorption equilibrium. Complete mathematical definition of system adsorption equilibria is difficult because of the above mentioned factors and also because of the large number of chemical species in the waste system. Information obtained toward this end, even if it is incomplete, is expected to be very helpful in understanding immobilization and/or conversely leachability mechanisms in the system. Waste species leachability (discussed in another section) is considered to be one of the most, if not the most, important criteria in determining the suitability of cement-based waste forms. A case can be made that leachability is a strong function of adsorption equilibria and of adsorption/desorption rates in the system.

Recognition that diffusion rates in high density materials are slower than in low density materials mitigates in favor of applying conditions to obtain high density waste-form products. Conversely, a low density product with high porosity and high internal surface is expected to exhibit a greater waste immobilization capacity. This problem needs further examination. The most beneficial system may well be one with a large pore volume and a large surface area with an intermediate level density but one in which the pore size distribution is strongly weighted toward smaller pores.

Continued fundamental research and new findings in, this area will almost certainly find quick application. 


\section{Solid phases}

ldeally, if all the phases in a given system are known, then thermodynamics may calculate the equilibrium state and quantify the driving forces. If the driving forces are quantified, then the rate constants may be quantified. From this information and given a state of the waste-form system, one could calculate where it wants to be (state at thermodynamic equilibrium) and how fast it will get there (kinetics). Even the cement paste alone may not be in well-ordered or crystalline phases, but cnough information could be gुenerated to approach this ideal.

Generally, the waste species are not likely to end up substituted into crystalline phases in the cement paste. Such substitutions would immobilize the species more than is generally observed. More fundamental work may help identify which species could be so immobilized and how to achieve this end. Usually, thermal treatments, as in making ceramics, would be required; but strontium, for example, might replace calcium in the calcium silicatc hydrates. Even so, soluble calcium species, e.g., calcium hydroxide, are still present in cement paste.

More likely, the waste species will be precipitated dispersed among the binder particles. The release of such species will be a combination of the continuous pore pathways to the waste-form surface, the surface area of the solid waste species exposed to the pore water, the solubility of the solid waste species, and the affinity of the soluble species for the matrix surface. Any one of these factors could be controlling.

Another likely possibility is that the waste species is bound or sorbed in the waste matrix which is heterogeneously dispersed in the cement matrix. For example, many organic fluids are hydrophobic and tend to accumulate on solid surfaces in sludges and soils. Depending on the mixing technique, these sludges and soils may he dispersed as micro-clumps throughout the cement matrix. This scenario presents a picture of mainly physical encapsulation of such sludges and soils. Additive agents may tend to sorb and retard the organic species that dissolve and diffuse through the porous matrix, but it is not well understood how accessible such agents are in the matrix. In other words, a sorptive agent that works well in the lab outside the matrix may be blocked and inaccessible in the matrix. Small changes in the matrix or the amount of additive might be the difference between success and faiiure. Treatment first with the additive prior to binding in the cement matrix may be the best approach, but some in situ remedial actions preclude this approach.

In addition, it is not always clear what effect the waste species has on the chemistry and microstructure of the cement matrix. Some waste species are known to retard or accelerate the set of the cement. The effect on the cement crystalline structure, gel structure, macropores, gel pores, and bound water is not necessarily clear. The use of pozzolans is said to provide active silica to react with the soluble calcium hydroxide to produce more calcium silicate hydrates in the pore structure; thus, producing lower porosities, more tortuous pathways, and lower matrix pHs. Granulated blast furnace 
slags are said to produce "lighter" matrices (meaning lower porosity and higher tortuosity) and a reducing matrix environment. It would be of benefit to establish these assertions definitively (or confirm that they have been definitively established).

\section{PERFORMANCE TESTS}

Generally, the performance test of most interest is leachability, i.e., the ability of the waste form to retain the waste species in a saturated aqueous environment. Other tests are used or specificd depending on the waste, application, matrix, elc., most often unconfined compressive strength (UCS). Usually the regulatory requirements for UCS are so low (300-400 $\mathrm{kPa}$ ) that binding into a monolith cannot be guaranted. In fact, some stabilization treatments leave a granulated product. Another factor becoming of more interest is the durability of the waste form or monolith. This usually means the structural integrity. In other words, do cracks form or does it break up into a granulated product by $x$ years? This would be of litle interest for granulated products, so the criteria and dependence on the physical barrier for retention determine the importance of durability.

\section{Leachability}

Although much time and effort hard teen expended in developing standard leach tests, there is still room for improvement. For cement-based waste forms, the standard test for radioactive wastes is the ANSI/ANS-16.1 leach iest, and the standaid test for hazardous wastes is the Toxicity Characteristic Leaching Procedure (TCLP).

The ANSI/ANS-16.1 test generates a single parameter (the leachability index) for assessing the leaching potential of a waste form and for comparative purposes among waste forms. The leachability index is generated from a simple diffusion madel, but the ANSI/ANS-16.1 procedure does not promise a model that will mimic the observed leaching thehavior, just a parameter that will be representative of the leaching behavior and can be used for comparative purposes. The leachability index accomplishes this purpose, quite often accurately depicts diffusion-controlled leaching behavior, and generally represents a conservative assessment of leaching potential even when the leaching behavior is obviously not simple diffusion control. It is well known that leaching from these porous solid bodies is not a simple diffusion process, even when the mathematics of diffusion accurately mimic the observed leaching behavior. The diffusion coefficients are a function of the true diffusivity of the waste species, the geometric complexity of the porous solid hody (i.e., the deviation of the diffusion path from an unobstructed straight line), and the affinity of the waste species for the surface of the cement matrix. Addition of one or more other 
phenomena can lead to more complex hehavior that can be modeled only by the addition of more parameters. The single parameter, leachability index, is a good compromise for comparative purposes and more complex models are available if needed. Understanding the fundamental chemistry and microstructure of these waste forms should lead to even better models based on the fundamental principles involved and lead to better predictions and extrapolations of performance. One problem with the ANSI/ANS-16.1 procedure is that it is labor intensive and does generate a lot of leachates for analysis and disposal.

The TCLP generates a single extract in an overnight extraction and, thus, does not have the same problem as the ANSI/ANS-16.1 test. The TCLP was designed to measure the species extractability from waste in a failed co-disposal scenario and is not really appropriate for measuring the effectiveness of solidified waste forms. The procedure requires the size reduction (to $<9.5 \mathrm{~mm}$ ) of any solidified waste form and eliminates any incentive to solidify the waste form. If the cement-based waste form is effective in retention as a granulated product, it will be that much more effective as a monolith product. In addition, a size of $9.5 \mathrm{~mm}$ is fairly significant and could lead to variation in results (i.e., equilibrium may not be achieved overnight as intended).

A reasonable alternative for cement-based waste forms is to adopt overnight leaching as is done in the TCLP as a screering test, but of the monolithic waste form and of the size-reduced waste form. The size reduction should be on the order of passing through a 200 mesh sieve $(<0.074 \mathrm{~mm}$ ). The granulated product would give a measure of the equilibrium between the extract and solid and the monolith could be used to give the leachability index for the screcning test. Once all but one waste form was eliminated, then the more extensive ANSI/ANS-16.1 test could be used to generate the rate constants reeded for extrapolation of the laboratory performance. This would eliminate dependence of the leachability index on the first time period and identify waste forms with time delay behavior. A more fundamental understanding of the chemistry and microstructure may eliminate some of the confusion about diffusion modeling and suggest more logical leaching procedures.

Another point of controversy has been what Jeachant or extractant to use. The ANSI/ANS-16.1 procedure sperifies deionized water, and the TCLP specifies a buffered acetic acid at a $\mathrm{pH}$ dependent on the alkalinity of the waste form (for cement-based waste forms this usually means a target pH of 3.0). Other leachants have been distilled water, tap water, site water, brine, sea water, acid rain, citric acid, and organic solvents. Each choice had a proponent and good reason for the choice. Deionized or distilled water appears fairly neutral, can he compared for different applications, and hears more resemblance to groundwater than some of the other choices. Site water would benchmark the performance for the water expected at the site (although the ionic composition can vary greatly). Acid rain is becoming more widespread globally and a significant database has been developed on its composition, but its nature may change considerably as it is absorbed into the ground. A more fundamental understanding of the chemistry and microstructure of the cement-hased waste forms may enable one to predict the expected 
performance in these different leachants or if any significant difference can be expected. Perhaps the performance for one leachant can be corrected for another with confidence, if enough is learned about the waste forms and the relative effects of the different leachants.

\section{Durability}

The importance of durability in this paper is related to the ability of the waste form 10 resist leaching rather than to bear a load. In general, even the most immobile waste form leaches, albeit the rate becomes vanishingly small. The rate of leaching is dependent on the surface area of the bulk waste form accessible to the bulk leachant and that is where durability becomes important. Over time periods of decades, centuries, and millennium, cement-based waste forms may crack and crumble, greatly increasing the area accessible to leaching. In addition, this act of weathering may change the nature of the waste form or how the waste species are retained. This possibility depends on learning more about the fundamental chemistry and microstructure of the waste forms, the increase in surface area is an effect that is already understood.

Besides the increased leaching area of the waste forms, durability of cement-based structures is also important because cement-based barriers are also used to prevent water intrusion into waste storage areas. If this physical barrier fails, it could result in environmental insult in some cases.

Engineers feel they understand the mechanisms of concrcte deterioration pretty well and have studied and modeled these mechanisms. In general, this work has not translated into predicting cracks or the increased surface area that is needed for risk assessment. At least one model has made an attempt to do this, but it is considered the first step and needs to be improved and benchmarked.

\section{SUMMARY}

This paper has suggested some of the research needs for cement-based waste forms. Definitive establishment of the correlation between some of the measurable physical properties of cement-based waste forms, such as porosity, and performance, such as leachability is needed. Even more important is a better fundamental understanding of the chemistry and microstructure of cement-based waste forms. With such understanding, not only can the performance of these waste forms be predicted and extrapolated hetter, but also these waste forms can he designed to perform heiter. In addition, better, cheaper feach tests are needed. Also, as confiuence grows in the ability to extrapolate leachability, the need will grow to better predict the durability of the waste form matrix. The challenge now is for everyone to add their lists of rescarch needs, openly debate which research needs to address with limited 
resources, and set about the task of proving where cement-based waste forms can solve some of the waste disposal problems of the future. 

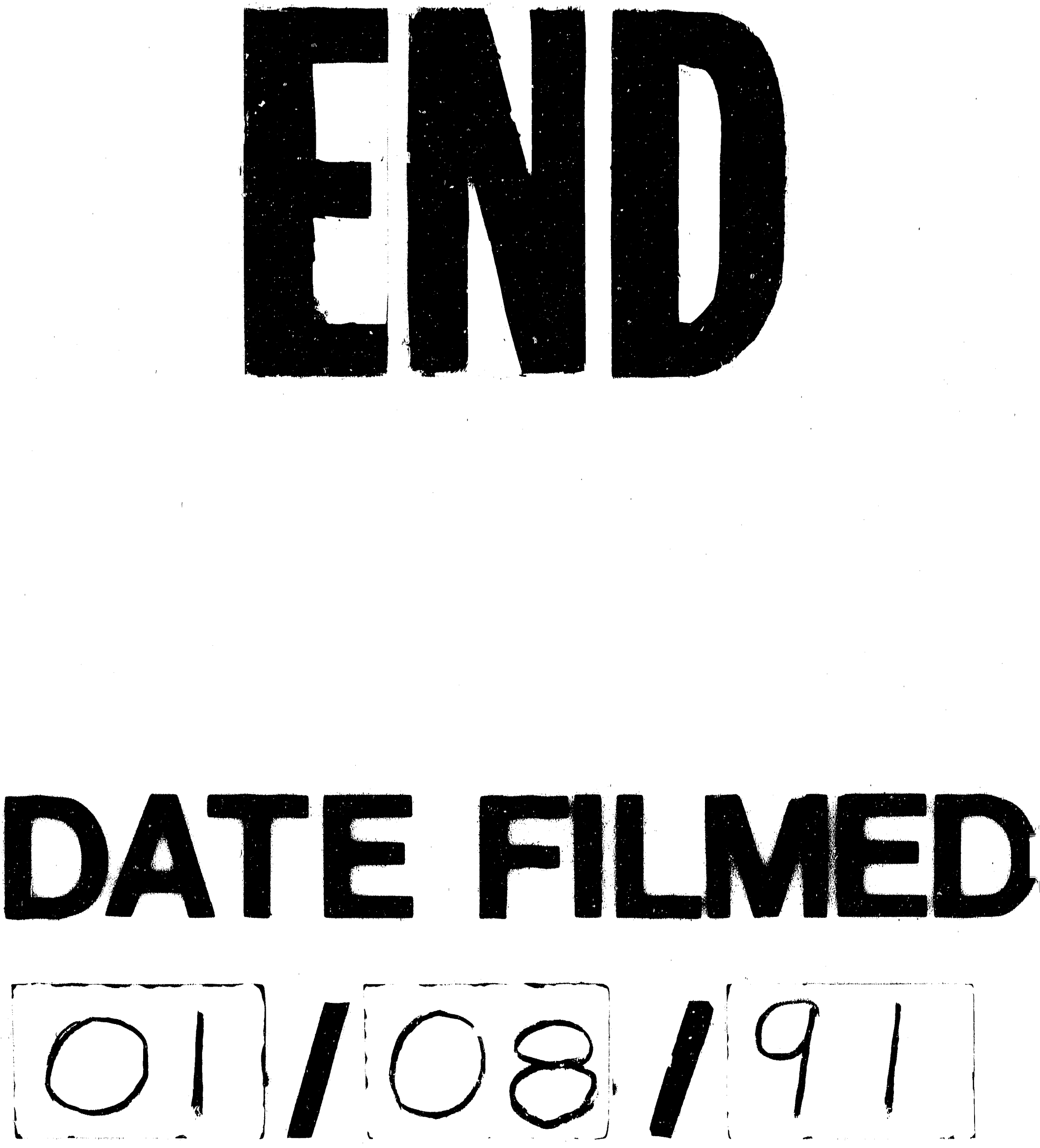
